\title{
Associations of Apolipoprotein A, High-Sensitivity C-Reactive Protein and Fasting Plasma Insulin in Obese Children With and Without Family History of Cardiovascular Disease
}

\author{
Zacharoula Karabouta $^{\text {a, c }}$, Dimitrios Papandreou ${ }^{\mathrm{a}, \mathrm{b}}$, Areti Makedou ${ }^{\mathrm{a}}$, Israel Rousso ${ }^{\mathrm{a}}$, \\ Fani Athanassiadou ${ }^{\mathrm{a}}$
}

\begin{abstract}
Background: The worldwide prevalence of childhood obesity has increased from $4.2 \%$ to $6.7 \%$ during the last two decades. Pediatric obesity is a major health problem, which is dramatically increasing in Greece. A variety of inflammatory variables have been also found to associate with cardiometabolic (CV) risk in obese children. The purpose of this study was to identify and examine the effects of possible $\mathrm{CV}$ risk factors in obese and non-obese children with and without family history $(\mathrm{FH})$ of cardiovascular disease (CVD).
\end{abstract}

Methods: Sixty-eight (68) healthy children and adolescents aged 7 - 13 years participated in the study. Anthropometrical and biochemical indexes were obtained from all children as well as $\mathrm{FH}$ of CVD.

Results: Systolic blood pressure (SBP), total cholesterol (TC), triglyceride (TG), high-sensitivity C-reactive protein (hsCRP), fasting plasma insulin (FPI) and homeostasis model assessment of insulin resistance (HOMA-IR) levels were found statistically significantly higher in the obese group compared to the non-obese one. High-density lipoprotein (HDL) levels were observed to be statistically significantly lower in the obese children compared to their normal peers.

Conclusions: Apolipoprotein A, hsCRP and FPI levels were significantly higher in the obese children with FH of CVD compared to the ones without FH of CVD. TC and SBP were found to be independently associated with obesity (odds ratio (OR): $1.965,95 \%$ confidence interval (CI): $1.935-2.97, \mathrm{P}<0.031$ and OR: $1.045,95 \% \mathrm{CI}: 1.016$ $-1.074, \mathrm{P}<0.002$, respectively).

Keywords: Obesity; Children; Cardiometabolic risk factors; Greece

Manuscript accepted for publication March 09, 2016

aThe 2nd Department of Pediatrics, Aristotle University of Thessaloniki, School of Medicine, University General Hospital of Thessaloniki AHEPA, Greece

bepartment of Natural Sciences \& Public Health, Zayed University, AbuDhabi, UAE

${ }^{\mathrm{c}}$ Corresponding Author: Zacharoula Karabouta, The 2nd Department of Pediatrics, University General Hospital of Thessaloniki AHEPA, St. Kiriakidis 1, P.O. 54636, Thessaloniki, Greece. Email: zkarabouta@yahoo.com

doi: http://dx.doi.org/10.14740/jocmr2531w

\section{Introduction}

The worldwide prevalence of childhood obesity has increased from $4.2 \%$ to $6.7 \%$ during the last two decades [1]. Data from 144 countries suggest that about 43 million children are overweight and obese and 92 millions are at risk of becoming obese later on their life [1].

Pediatric obesity is a major health problem and is dramatically increasing in Greece $[2,3]$. Consequences such as hypertension, type 2 diabetes (T2D), non-alcoholic fatty liver disease (NAFLD), hyperlipidemia, hyperglycemia and insulin resistance (IR) are serious health issues that have been found to be established at young ages in pediatric population in Greece [4-6].

Cardiovascular (CV) risk in children becomes significant from the 85th percentile for weight and above where other risk factors changes are observed, such as high-density lipoprotein (HDL), low-density lipoprotein (LDL), triglyceride (TG) and total cholesterol (TC) [7]. Atherosclerosis is a process that starts in early life as Berenson et al have reported, body mass index (BMI), high blood pressure (BP) and high concentrations of LDL, TC and low HDL were strongly associated with the extension of lesions in the coronary arteries [8].

A variety of inflammatory variables have been also found to be associated with CV risk in obese children. Kim et al [9] observed in 354 children, that the obese group had higher levels of high-sensitivity C-reactive protein (hsCRP), interleukin and tumor necrosis factor- $\alpha$ compared to the non-obese ones.

IR is another well-known CV risk factor in adults and has been proved to have a very strong association with pediatric obesity [10]. In addition, metabolic syndrome, which is basically a cluster of $\mathrm{CV}$ risk factors such as hyperinsulinemia, obesity, hypertension and hyperlipidemia, has been observed in obese children [6].

As biomarkers of lipid metabolism, apolipoprotein A (ApoA) and apolipoprotein B (ApoB) have been found to be good predictors for cardiovascular disease (CVD) [11]. In addition, lipoprotein(a) (Lp(a)) which is mainly produced by monocytes/macrophages and primarily bound to LDL cholesterol in the peripheral circulation, was found to be predictive for $\mathrm{CV}$ events in adults [12].

Finally, family history (FH) is another important and independent $\mathrm{CV}$ risk factor, especially for early-onset disease. Many studies have shown a two- to three-fold increase in CVD 
given a first-degree relative with CVD and the strength of this association increases as the number of affected first-degree relatives increases $[13,14]$.

The purpose of this study was to identify and examine the effects of possible CV risk factors in obese and non-obese children with and without FH of CVD.

\section{Materials and Methods}

Sixty-eight (68) children and adolescents aged 7 - 13 years (35 obese and 33 controls) participated in the study. All subjects were recruited from a pediatric obesity outpatient's clinic and a clinic for lipids and prevention of CVD in childhood. The power calculation to have the appropriate number to contact the study was 32 children. Controls were healthy children and adolescents, with normal BMI. A detailed medical history was taken of each child by a pediatrician of our clinic. Children with clinical evidence of diabetes, $\mathrm{CV}$, liver disease and use of alcohol, drugs or other disease were excluded from the study. FHs of CVD, hypertension and hyperlipidemia were also obtained from each subject. The latter was defined as positive if there was a first-degree male relative (father or grandfather) diagnosed with CVD under the age of 55 or a first-degree female relative (mother or grandmother) diagnosed with CVD under the age of 65 . The term CVD includes all the diseases of the heart and circulation including coronary heart disease (angina and heart attack), heart failure, congenital heart disease and stroke. The Ethical Committee of the Aristotle University of Thessaloniki approved the study and a written informed consent form was also obtained from the children's guardians who participated in the study.

\section{Anthropometrical indices}

Anthropometrical indices such as height, weight, Tanner stage and waist circumference were measured in all children. Body weight was measured to the nearest $(0.1 \mathrm{~kg})$ wearing light clothes and without shoes using SECA 700 model-scale. Height was measured to the nearest $(0.1 \mathrm{~cm})$ using a SECA stadiometer. Obesity was defined by the new revised international child BMI cut-offs for sex, according to the World Obesity Federation (previously IOTF) [15]. Blood pressure was measured by auscultation using a mercury sphygmomanometer. The subjects were in seated position, with arm flexed at the level of the heart. A properly sized BP cuff for children was used to measure BP. The length of the cuff's bladder was at least equal to $80 \%$ of the circumference of the upper arm. The BP was measured three times and the mean average was considered to be the overall BP value. Normal systolic BP was defined when percentile was $<90$ th percentile, pre-hypertensive when percentile $\geq 90$ th and $<95$ th and hypertensive was defined as either systolic and/or diastolic BP $\geq 95$ th percentile measured upon three or more occasions. Children and adolescents whose BP exceeded 120/80 mm Hg were considered pre-hypertensive even if the blood pressure was $<90$ th percentile [16].
Table 1. Basic Characteristics Between Boys and Girls $(n=$ 68)

\begin{tabular}{llll} 
& $\begin{array}{l}\text { Boys }(\mathbf{N}=\mathbf{2 8}), \\
\text { mean } \pm \text { SD }\end{array}$ & $\begin{array}{l}\text { Girls }(\mathbf{N}=\mathbf{4 0}), \\
\text { mean } \pm \text { SD }\end{array}$ & P-value \\
\hline Age $($ years $)$ & $9.78 \pm 3.48$ & $9.48 \pm 2.71$ & 0.684 \\
Height $(\mathrm{cm})$ & $1.41 \pm 0.19$ & $1.39 \pm 0.15$ & 0.479 \\
\hline Weight $(\mathrm{kg})^{\mathrm{a}}$ & $50.2 \pm 26.4$ & $43 \pm 21.9$ & 0.223 \\
BMI $\left(\mathrm{kg} / \mathrm{m}^{2}\right)^{\mathrm{a}}$ & $23.67 \pm 7.4$ & $21.2 \pm 6.6$ & 0.182 \\
SBP $(\mathrm{mm} \mathrm{Hg})$ & $59.72 \pm 7.8$ & $50.3 \pm 29.2$ & 0.185 \\
DBP $(\mathrm{mm} \mathrm{Hg})$ & $57.32 \pm 8.8$ & $49.2 \pm 25.4$ & 0.224 \\
TC $(\mathrm{mg} / \mathrm{dL})^{\mathrm{a}}$ & $162.4 \pm 45.5$ & $162.8 \pm 29.7$ & 0.974 \\
TG $(\mathrm{mg} / \mathrm{dL})^{\mathrm{a}}$ & $78.5 \pm 39.5$ & $83.9 \pm 45$ & 0.605 \\
HDL $(\mathrm{mg} / \mathrm{dL})^{\mathrm{a}}$ & $48.3 \pm 14.7$ & $52.3 \pm 16.8$ & 0.307 \\
LDL $(\mathrm{mg} / \mathrm{dL})$ & $92 \pm 24.1$ & $95 \pm 22.8$ & 0.543 \\
ApoA $(\mathrm{mg} / \mathrm{dL})$ & $133.53 \pm 0$ & $140.3 \pm 27.1$ & 0.330 \\
ApoB $(\mathrm{mg} / \mathrm{dL})^{\mathrm{a}}$ & $69.9 \pm 22.2$ & $63.6 \pm 15.1$ & 0.165 \\
\hline
\end{tabular}

$\mathrm{P}<0.05$. aMann-Whitney test, all other $t$-test.

\section{Biochemical indices}

A blood sample of $5 \mathrm{~mL}$ was drawn by venipuncture after the subjects had fasted overnight for $10 \mathrm{~h}$. Fasting serum TC, HDLcholesterol and TGs were ascertained with direct measurement assays using standard laboratory methods (Roche Diagnostics) while LDL-cholesterol was calculated using Friedwald's equation. hsCRP levels were determined using particle enhanced immunoturbidimetric method. ApoA and B were measured by the immunoturbidimetric method. Fasting blood glucose and serum insulin levels were assessed by enzymatic method with hexokinase and electrochemiluminescence immunoassay "ECLIA", respectively. IR was calculated by means of the homeostasis model assessment (HOMA) with the HOMA calculator (University of Oxford, Diabetes Trials Unit, the Oxford Centre for Diabetes, Endocrinology and Metabolism). IR was defined by HOMA $>2.5 \mathrm{IU} / \mathrm{mL}$.

\section{Statistics}

The Shapiro-Wilk statistic was used to test the normality assumption of the numerical variables of the study. The main effect of the group (normal, obese) of FH of CVD (positive, negative) and their interaction effect were assessed with twoway ANOVA for normally distributed variables and with Kruskal-Wallis test for non-normally distributed data. In case of ANOVA, pairwise comparisons were conducted with Bonferroni method, while Mann-Whitney U test with Bonferroni adjusted P-values was used for pairwise comparisons after the Kruskal-Wallis test. Comparisons between boys and girls were done with independent samples $t$-test (normally distributed data) or with Mann-Whitney U test (non-normally distributed data). Univariate and multivariate logistic regression models were used to associate systolic BP, TC, TG, HDL, ApoA, 
Table 2. Comparison of Normal and Obese Groups With and Without FH of CVD

\begin{tabular}{|c|c|c|c|c|c|c|}
\hline \multirow{2}{*}{ Variables } & \multicolumn{3}{|c|}{ Normal } & \multicolumn{3}{|c|}{ Obese } \\
\hline & PFH $(n=8)$ & NFH $(n=25)$ & Total $(n=33)$ & PFH $(n=15)$ & NFH $(n=20)$ & Total $(n=35)$ \\
\hline SBP & $54 \pm 23.9$ & $38.9 \pm 26.6$ & $42.5 \pm 26.4$ & $68.5 \pm 24.9$ & $63.2 \pm 28.2$ & $65.5 \pm 26.6^{*}$ \\
\hline DBP & $47.5 \pm 30.1$ & $51.2 \pm 30.4$ & $50.3 \pm 29.9$ & $53.3 \pm 29.9$ & $56 \pm 19.9$ & $54.9 \pm 24.3$ \\
\hline $\mathrm{TC}$ & $167 \pm 33$ & $166 \pm 30$ & $167 \pm 31$ & $191 \pm 18$ & $178 \pm 24$ & $181 \pm 24.5^{*}$ \\
\hline TG & $59.5 \pm 22.5$ & $73.8 \pm 42.2$ & $70.4 \pm 38.7$ & $88 \pm 44.5$ & $95.9 \pm 44.2$ & $92.5 \pm 43.8^{*}$ \\
\hline HDL & $64.9 \pm 19.3$ & $54.4 \pm 17.5$ & $56.9 \pm 18.2$ & $41.9 \pm 10$ & $46.5 \pm 10.6$ & $44.5 \pm 10.5^{*}$ \\
\hline LDL & $88.8 \pm 29.5$ & $92 \pm 43.4$ & $90.4 \pm 40.6$ & $104.4 \pm 21$ & $99.5 \pm 23$ & $101 \pm 22.9$ \\
\hline ApoA & $159.9 \pm 25$ & $141 \pm 31.6$ & $145.6 \pm 31$ & $135.4 \pm 21.8^{* *}$ & $121 \pm 23.6$ & $129 \pm 23.3^{*}$ \\
\hline ApoB & $61 \pm 15.6$ & $67.3 \pm 19.3$ & $65.9 \pm 18.5$ & $66.6 \pm 22.8$ & $66.7 \pm 15.9$ & $66.7 \pm 18.9$ \\
\hline $\operatorname{Lp}(\mathrm{a})^{\mathrm{a}}$ & $20(3.4-113.9)$ & $21.2(2-200)$ & $21.2(2-200)$ & $16.5(4.1-106)$ & $16.5(4.4-87)$ & $16.5(4.1-106)$ \\
\hline hsCRPa & $0.11(0.01-2.54)$ & $0.08(0.01-2.6)$ & $0.08(0.01-2.6)$ & $0.78(0.03-2.8)$ & $0.73(0.02-2.7)$ & $0.75(0.02-2.8)^{*}$ \\
\hline FBG & $84.4 \pm 12$ & $88 \pm 7.7$ & $87 \pm 8.9$ & $87 \pm 8.7$ & $89.5 \pm 8$ & $88.4 \pm 8.4$ \\
\hline $\mathrm{FPI}^{\mathrm{a}}$ & $6.6(1.1-35.6)$ & $8.6(2.9-159)$ & $8.2(1.1-159)$ & $16(4.5-42)^{* *}$ & $11.8(0.2-31)$ & $12.8(0.2-42)^{*}$ \\
\hline HOMA-IR ${ }^{b}$ & $\begin{array}{l}0.95 \times 2.28 \\
(0.35-4.35)\end{array}$ & $\begin{array}{l}1.06 \times 1.68 \\
(0.38-3.24)\end{array}$ & $\begin{array}{l}1.03 \times 1.81 \\
(0.35-4.35)\end{array}$ & $\begin{array}{l}1.8 \times 1.9 \\
(0.58-5.24)\end{array}$ & $\begin{array}{l}1.43 \times 1.77 \\
(0.37-3.83)\end{array}$ & $\begin{array}{l}1.58 \times 1.83 * \\
(0.37-5.24)\end{array}$ \\
\hline
\end{tabular}

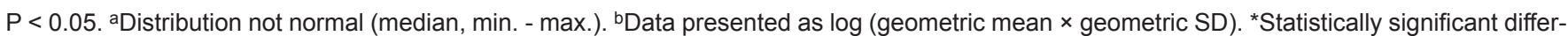
ence between obese and normal group. ${ }^{* *}$ Statistically significant difference between obese and normal group with PFH.

hsCRP, fasting insulin $0^{\prime}$ and HOMA-IR with group (normal, obese) and FH of CVD (positive, negative). Results of those models included odds ratios (OR), 95\% confidence intervals (CIs), sensitivity and specificity. The overall analysis was performed with IBM Statistics SPSS 20.0 and the statistical significance was set at $\mathrm{P}<0.05$.

\section{Results}

Out of the 68 children, 35 were obese and 33 non-obese. Fifteen (15) out of the 35 obese ones had positive FH of CVD while eight children out of the 33 non-obese ones had positive FH of CVD.

Table 1 represents the anthropometrical and biochemical profile of all children based on gender. No statistically significant differences were observed between boys and girls.

SBP, TC, TG, hsCRP, fasting plasma insulin (FPI) and HOMA-IR levels were found statistically significantly higher in the obese group compared to the normal one. Additionally, HDL and ApoA levels were observed to be statistically significantly lower in the obese children compared to their normal peers. Moreover, ApoA, hsCRP and FPI levels were significantly higher in the obese children with FH of CVD compared to those without FH of CVD (Table 2).

Figure 1 depicts the strong positive relation of FPI with HOMA-IR $(\mathrm{r}=0.998, \mathrm{P}<0.001)$.

In a univariate logistic regression model, none of the $\mathrm{CV}$ risk factors were related to children with and without $\mathrm{FH}$ of CVD (Table 3).

Table 4 represents the OR of children having obesity to the normal ones. Obese children have almost double risk of having high TC levels (OR: 1.965 95\% CI: 1.935 - 2.970, P
$<0.031$ ), while for the SBP the risk was OR: $1.045,95 \%$ CI: $1.016-1.074, \mathrm{P}<0.002$.

\section{Discussion}

In this study, we attempt to identify any possible relation of $\mathrm{CV}$ risk factors in 68 obese and non-obese children with and without FH of CVD.

In our study, obese children were found to have higher risk of abnormal total fasting serum cholesterol and systolic BP levels compared to normal children. Many authors have evaluated the relationship of childhood obesity with BP. A European pediatric cohort showed that high BP was seen in about $35 \%$ of overweight children [17]. In another recent study [18] that covered 26,000 school children, the systolic hypertension observed was $5.3 \%, 12.3 \%$ and $14.6 \%$ for normal, overweight and obese children, respectively. High levels of hypertension reported in other studies from Greece should be under serious concern. Angelopoulos et al [19] examined 312 children aged 11 years and found prevalence rates of $29.4 \%$ and $11.8 \%$ for boys and $39 \%$ and $7.5 \%$ for girls. Additionally, others examined 606 children from Northern Greece and reported a prevalence of systolic and diastolic hypertension of $12.3 \%$ and $15.1 \%$ for boys and $13.3 \%$ and $15.1 \%$ for girls, respectively [20]. The probable mechanism of obesity-related hypertension might include IR, sodium retention, increased sympathetic nervous system activity and other factors [21].

Obesity is commonly associated with a combined dyslipidemia pattern with mild elevation in TC and LDL-cholesterol levels, moderate-to-severe elevation in TG level, and low HDL-cholesterol levels [22]. In our study, the obese group presented significantly higher levels of TC, LDL and TG and 


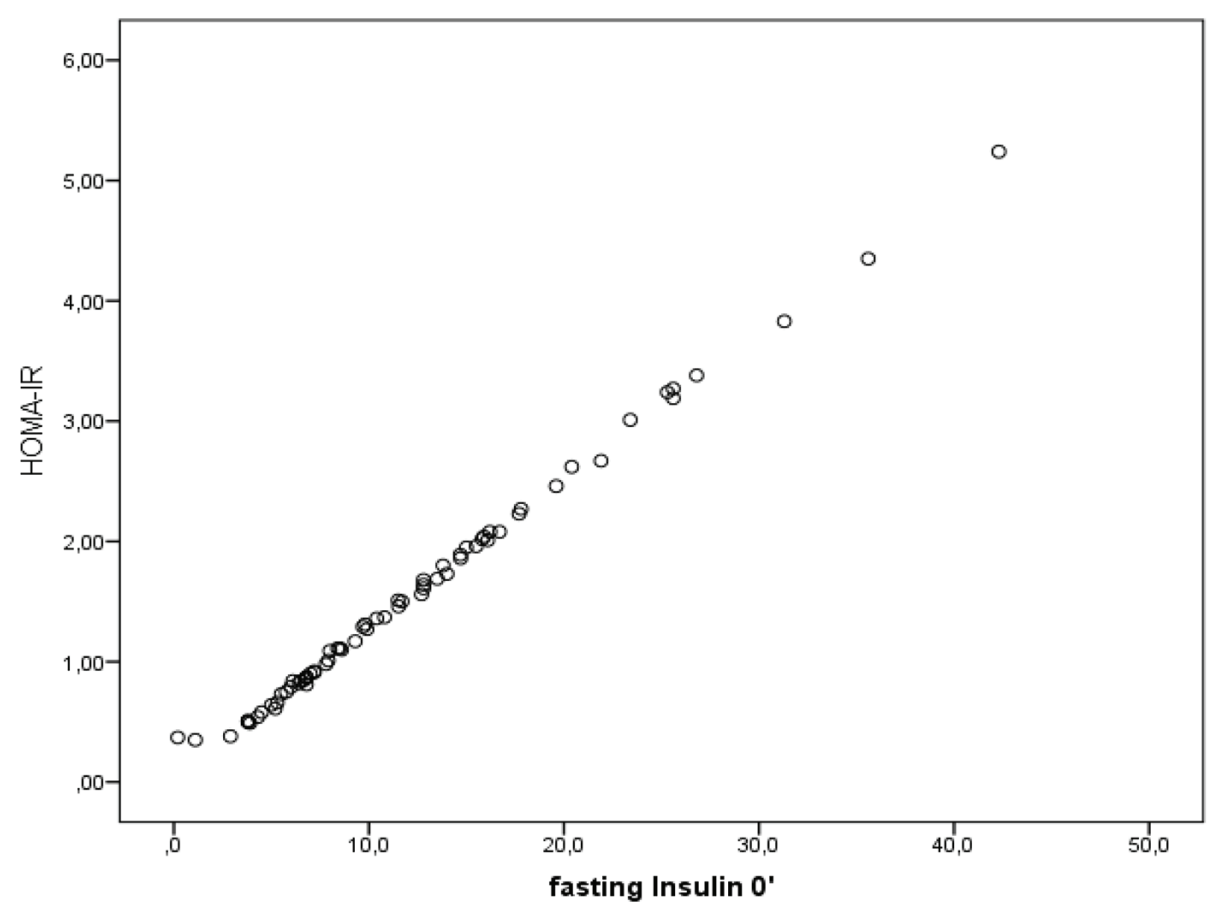

Figure 1. Relation of HOMA with IR $(r=0.998, P<0.001)$.

lower levels of HDL compared to the non-obese group. In the multiple regression model though, only TC was independently associated with obesity. Weight loss should be the first priority in order to reduce TC and systolic BP in obese children. A few studies on children based on short-term weight loss over a few weeks showed an improvement of CV risk factor profile associated with obesity (reduction of hypertension, TG, and TC) $[23,24]$. Additionally, a diet with total fat at $25-30 \%$ of calories, saturated fat at $<10 \%$ of calories, and cholesterol intake at $<300 \mathrm{mg} / \mathrm{day}$, as recommended by the original NCEP Pediatric Panel [25] has been shown to safely and effectively reduce the levels of TC and LDL-cholesterol in healthy children.

Table 3. Univariate Logistic Regression Analysis Between Cardiometabolic Risk Factor and Family History of CVD

\begin{tabular}{lllll}
\hline & \multicolumn{4}{c}{ Univariate logistic regression results* } \\
\cline { 2 - 5 } & OR & $\mathbf{9 5 \%}$ CI & P-value & $\begin{array}{l}\text { Sensitivity/ } \\
\text { specificity }\end{array}$ \\
\hline SBP (mm Hg) & 1.018 & $0.999-1.037$ & 0.064 & $0.13 / 0.93$ \\
TC (mg/dL) & 1.983 & $1.965-2.001$ & 0.070 & $0.13 / 0.96$ \\
TG (mg/dL) & 0.997 & $0.985-1.009$ & 0.624 & $0.00 / 1.00$ \\
HDL (mg/dL) & 0.996 & $0.965-1.028$ & 0.801 & $0.00 / 1.00$ \\
ApoA (mg/dL) & 0.995 & $0.978-1.013$ & 0.603 & $0.00 / 1.00$ \\
hsCRP & 1.367 & $0.689-2.715$ & 0.371 & $0.00 / 1.00$ \\
FPI (mIU/mL) & 1.058 & $0.993-1.127$ & 0.080 & $0.17 / 0.94$ \\
HOMA-IR & 1.570 & $0.941-2.619$ & 0.084 & $0.17 / 0.94$ \\
\hline
\end{tabular}

${ }^{*} \mathrm{P}<0.05$.
The paradox in our study is that we did not find any relation in the logistic regression model, between HOMA and obesity or FH of CVD. This is possibly due to the effect of the small number of subjects included in the study. However, these results should not underestimate the significance of HOMAIR, since IR is the main cause of hypertriglyceridemia, hyperlipidemia, decrease of HDL-cholesterol, and increased BP in obesity, and correlates to the degree of overweight [26]. Our study has its limitations, since information on genetics, physical activity, socioeconomic status and diet was not assessed. Additionally, the small sample size also might affect some of the cardiometabolic risk factors related to obese children especially those with FH of CVD.

Table 4. Logistic Regression Analysis Between Cardiometabolic Risk Factor and Obesity

\begin{tabular}{lllll}
\hline & \multicolumn{4}{l}{ Multivariate logistic regression analysis } \\
\cline { 2 - 5 } & OR & $\mathbf{9 5 \%}$ CI & P-value & $\begin{array}{l}\text { Sensitivity/ } \\
\text { specificity }\end{array}$ \\
\hline SBP (mm Hg) & 1.045 & $1.016-1.074$ & $0.002 *$ & $0.13 / 0.93$ \\
TC (mg/dL) & 1.965 & $1.935-2.970$ & $0.031^{*}$ & $0.13 / 0.96$ \\
TG (mg/dL) & 0.997 & $0.985-1.009$ & 0.624 & $0.00 / 1.00$ \\
HDL (mg/dL) & 0.996 & $0.965-1.028$ & 0.801 & $0.00 / 1.00$ \\
ApoA (mg/dL) & 0.995 & $0.978-1.013$ & 0.603 & $0.00 / 1.00$ \\
hsCRP & 2.92 & $0.966-8.828$ & 0.058 & $0.00 / 1.00$ \\
FPI (mIU/mL) & 1.058 & $0.993-1.127$ & 0.080 & $0.17 / 0.94$ \\
HOMA-IR & 1.570 & $0.941-2.619$ & 0.084 & $0.17 / 0.94$ \\
\hline
\end{tabular}

${ }^{*} \mathrm{P}<0.05$. 


\section{Conclusions}

ApoA, hsCRP and FPI levels were significantly higher in the obese children with FH of CVD compared to the ones without FH of CVD. TC and systolic BP were found to be independently associated with obesity. In contrast, FH of CVD was not related to any cardiometabolic risk factors. Prevention of obesity may decrease development of CV risk.

\section{Conflict of Interest}

None.

\section{Abbreviations}

BMI: body mass index; SBP: systolic blood pressure; DBP: diastolic blood pressure; TC: total cholesterol; TG: triglyceride; HDL: high-density lipoprotein; LDL: low-density lipoprotein; PFH: positive family history of CVD; NFH: negative family history of CVD; FBG: fasting blood glucose; FPI: fasting plasma insulin; ApoA: Apolipoprotein A; ApoB: Apolipoprotein B; CVD: cardiovascular disease; CV: cardiovascular; HOMA: homeostasis model assessment; IR: insulin resistance; NAFLD: non-alcoholic fatty liver disease

\section{References}

1. de Onis M, Blossner M, Borghi E. Global prevalence and trends of overweight and obesity among preschool children. Am J Clin Nutr. 2010;92(5):1257-1264.

2. Kapantais E, Haralambides V, Tzotzas T, et al. First National Epidemiological Large Scale Survey on the Prevalence of Childhood and Adolescent Obesity in Greece. Int J Obes. 2000;28(Suppl.71).

3. Magkos F, Manios Y, Christakis G, Kafatos AG. Age-dependent changes in body size of Greek boys from 1982 to 2002. Obesity (Silver Spring). 2006;14(2):289-294.

4. Papandreou D, Karabouta Z, Pantoleon A, Rousso I. Investigation of anthropometric, biochemical and dietary parameters of obese children with and without non-alcoholic fatty liver disease. Appetite. 2012;59(3):939-944.

5. Manios Y, Yiannakouris N, Papoutsakis C, Moschonis G, Magkos F, Skenderi K, Zampelas A. Behavioral and physiological indices related to BMI in a cohort of primary schoolchildren in Greece. Am J Hum Biol. 2004;16(6):639-647.

6. Papandreou D, Karabouta Z, Pantoleon A, Sidiropoulou A, Rousso I. Cardiometabolic risk factors related to nonalcoholic fatty liver disease in obese children. Experimental \& Clincal Cardiology. 2014;8(20):2194-2205.

7. Freedman DS, Dietz WH, Srinivasan SR, Berenson GS. The relation of overweight to cardiovascular risk factors among children and adolescents: the Bogalusa Heart Study. Pediatrics. 1999;103(6 Pt 1):1175-1182.

8. Berenson GS, Srinivasan SR, Bao W, Newman WP, 3rd,
Tracy RE, Wattigney WA. Association between multiple cardiovascular risk factors and atherosclerosis in children and young adults. The Bogalusa Heart Study. N Engl J Med. 1998;338(23):1650-1656.

9. Kim J, Bhattacharjee R, Kheirandish-Gozal L, Khalyfa A, Sans Capdevila O, Tauman R, Gozal D. Insulin sensitivity, serum lipids, and systemic inflammatory markers in school-aged obese and nonobese children. Int $\mathrm{J}$ Pediatr. 2010;2010:846098.

10. Kurtoglu S, Hatipoglu N, Mazicioglu M, Kendirici M, Keskin M, Kondolot M. Insulin resistance in obese children and adolescents: HOMA-IR cut-off levels in the prepubertal and pubertal periods. J Clin Res Pediatr Endocrinol. 2010;2(3):100-106.

11. Thompson A, Danesh J. Associations between apolipoprotein B, apolipoprotein AI, the apolipoprotein B/ AI ratio and coronary heart disease: a literature-based meta-analysis of prospective studies. J Intern Med. 2006;259(5):481-492.

12. Koenig W, Twardella D, Brenner H, Rothenbacher D. Lipoprotein-associated phospholipase A2 predicts future cardiovascular events in patients with coronary heart disease independently of traditional risk factors, markers of inflammation, renal function, and hemodynamic stress. Arterioscler Thromb Vasc Biol. 2006;26(7):1586-1593.

13. Slack J, Evans KA. The increased risk of death from ischaemic heart disease in first degree relatives of 121 men and 96 women with ischaemic heart disease. J Med Genet. $1966 ; 3(4): 239-257$.

14. Hunt SC, Williams RR, Barlow GK. A comparison of positive family history definitions for defining risk of future disease. J Chronic Dis. 1986;39(10):809-821.

15. Cole TJ, Lobstein T. Extended international (IOTF) body mass index cut-offs for thinness, overweight and obesity. Pediatr Obes. 2012;7(4):284-294.

16. The fourth report on the diagnosis, evaluation, and treatment of high blood pressure in children and adolescents. Pediatrics. 2004;114(2 Suppl 4th Report):555-576.

17. I'Allemand D, Wiegand S, Reinehr T, Muller J, Wabitsch M, Widhalm K, Holl R. Cardiovascular risk in 26,008 European overweight children as established by a multicenter database. Obesity (Silver Spring). 2008;16(7):16721679.

18. Raj M, Sundaram KR, Paul M, Deepa AS, Kumar RK. Obesity in Indian children: time trends and relationship with hypertension. Natl Med J India. 2007;20(6):288-293.

19. Angelopoulos PD, Milionis HJ, Moschonis G, Manios Y. Relations between obesity and hypertension: preliminary data from a cross-sectional study in primary schoolchildren: the children study. Eur J Clin Nutr. 2006;60(10):1226-1234.

20. Papandreou D, Stamou M, Malindretos P, Rousso I, Mavromichalis I. Prevalence of hypertension and association of dietary mineral intake with blood pressure in healthy schoolchildren from northern Greece aged 7-15 years. Ann Nutr Metab. 2007;51(5):471-476.

21. Kotchen TA. Obesity-related hypertension: epidemiology, pathophysiology, and clinical management. Am J Hypertens. 2010;23(11):1170-1178. 
22. Expert panel on integrated guidelines for cardiovascular health and risk reduction in children and adolescents: summary report. Pediatrics. 2011;128(Suppl 5):S213256.

23. Sothern MS, Despinasse B, Brown R, Suskind RM, Udall JN, Jr., Blecker U. Lipid profiles of obese children and adolescents before and after significant weight loss: differences according to sex. South Med J. 2000;93(3):278282.

24. Epstein LH, Kuller LH, Wing RR, Valoski A, McCurley
J. The effect of weight control on lipid changes in obese children. Am J Dis Child. 1989;143(4):454-457.

25. National Cholesterol Education Program (NCEP): highlights of the report of the Expert Panel on Blood Cholesterol Levels in Children and Adolescents. Pediatrics. 1992;89(3):495-501.

26. Isomaa B, Almgren P, Tuomi T, Forsen B, Lahti K, Nissen M, Taskinen MR, et al. Cardiovascular morbidity and mortality associated with the metabolic syndrome. Diabetes Care. 2001;24(4):683-689. 